JURNAL RESPIRASI

JR

Vol. 6 No. 1 January 2020

\title{
Thrombocytopenia in Young Patient due to Anti Tuberculosis Drugs : A Case Report
}

\author{
Aryani Prawita Sari*, Winariani Koesoemaprodjo \\ Departement of Pulmonology and Respiratory Medicine, Faculty of Medicine, Universitas Airlangga - Dr. Soetomo General \\ Hospital, Surabaya
}

\begin{abstract}
Background: Most anti-tuberculosis (ATD) drugs are relatively safe, but unusual serious reactions can occur. Thrombocytopenia is an uncommon but potentially life-threatening complication of certain ATDs and is characterized by rapid destruction of platelets whenever an offending drug is taken by a susceptible person. Rifampicin is the most common cause of thrombocytopenia. Case: A 25 years old woman came with chief complaints, shortness of breath since 1 week before admission and cough with phlegm since 2 months before admission. The patient received antibiotic and ATD. In the course of improving on sepsis and pneumonia, the patient had thrombocytopenia accompanied by melena on day 4 of treatment. Discussion: Thrombocytopenia is defined as a disorder, which showed an abnormality on the low amount of thrombocyte. Thrombocytopenia was commonly cofounded when Complete blood count (CBC) was performed. The majority of the mechanism associated with thrombocytopenia is the immune. Drug-induced Thrombocytopenia (DITP) is an exclusion diagnosis, which is obtained by ruling out other underlying causes that resulted in thrombocytopenia Conclusion: This case illustrates that the discovery of isolated thrombocytopenia in a patient taking several medications presents a challenging clinical problem. Laboratory confirmation of druginduced thrombocytopenia at the time of initial presentation is not possible because tests for drug-dependent anti-platelet antibodies are not available in most clinical laboratories. The diagnosis of drug-induced thrombocytopenia can be supported only by resolution of thrombocytopenia after discontinuation of therapy with the suspected drug.
\end{abstract}

Keywords: anti tuberculosis drugs, pulmonary tuberculosis, thrombocytopenia

Correspondence: Aryani Prawita Sari, Departement of Pulmonology and Respiratory Medicine, Faculty of Medicine, Universitas Airlangga/Dr. Soetomo General Hospital, Surabaya, Indonesia. Jl. Mayjen Prof. Dr. Moestopo 6-8 Surabaya. Email: aryani_sari@yahoo.com

\section{INTRODUCTION}

Tuberculosis (TB) is a global health problem. Immigration and increased incidence of this disease in the younger population has generated a significant shift in the epidemiology of the disease. Additionally, the escalated incidence of TB in epidemic areas had been reported as a deflation. TB infection and latent TB infection (LTBI) in women of childbearing age has yielded a diagnostic and therapeutic challenge, current management evaluation and recommendation are needed as a strategy to control the disease in the future. ${ }^{1}$

TB treatment has been a therapeutic challenge for a long time. Many of anti-tuberculosis drug (ATD) is relatively safe, yet serious and unusual adverse reaction could happen. Thrombocytopenia is one of the rare adverse effects, but potentially lethal that could occur in some ATD. Rifampicin is an ATD that frequently develops thrombocytopenia, as well as Isoniazid and Pyrazinamide. Identifying the underlying cause of thrombocytopenia in a patient, who undergoes multidrug therapy, has yielded a clinical problem. Confirmation of drug-induced thrombocytopenia in the early treatment is infrequently prompted, which is a drug-dependent antibody anti-platelet, a test that is unavailable in many major laboratories. If discontinuation of the suspected drug has resulted in improved thrombocytopenia, then it is strongly advised that thrombocytopenia was induced by drug administration. ${ }^{2,3}$

Rifampicin-induced thrombocytopenia was first reported in 1970. This process is still reversible if it is detected and treated correctly. The other drugs that have been widely known inducing thrombocytopenia are Quinine, Quinidine, Chloroquine, Sulfonamide, Tolbutamide, Chlorothiazide, Digoxin, Penicillamine, Amphotericin B, sedative agent, anticonvulsant, Metildopa, and Aspirin., ${ }^{2,3}$

This study is a case report of a woman, who came with shortness of breath, that was further diagnosed as pulmonary TB and received category one of ATD. In her progress, hrombocytopenia was developed and lead to bleeding manifestation as melena. 


\section{CASE}

A patient was admitted to Dr. Soetomo General Hospital on July 13th, 2016, with chief complaint as shortness of breath. This complaint had occurred for one week and worsened two days before admission. Productive cough had appeared since two months before admission with a change of sputum from white to yellowish one week before admission. The patient also mentioned a subfebrile condition during this period. There were also enlargement of lymph nodes in the left and right side of the neck that was known two days before admission. These enlargements had not been examined before. There were also loss of appetite, night sweats, and unintentional weight loss. The patients were 22 days following after giving birth when admitted, and puerperal bleeding still occurred.

Physical examination showed four enlargements of lymph nodes in the right and left side of the neck, sized $\pm 1.5 \mathrm{~cm} \times 1.2 \mathrm{~cm} \times 1 \mathrm{~cm}$, without any tenderness. Vocal fremitus was decreased in 1/3 lower of right and left lungs. In auscultation, there was diminished vesicular breath sound in $1 / 3$ lower of right and left lungs.

\section{Chest Radiology}

Base on chest radiology on July 13th, 2016 (figure 1), bilateral pleural effusions and infiltrates in both lungs was found. Pleural punction was conducted on left hemithorax and collected $150 \mathrm{~mL}$ of pleural fluid. Consultation from Department of Obstetric Gynecology showed P3003 postpartum spontaneous vaginal delivery day 22 without any abnormality. Consultation with Department of Cardiology mentioned cardiomyopathy peripartum diagnosis could not be ruled out yet, with suggestion to conduct Echocardiography. Then, consultation with Department of Internal Medicine had provided diagnosis anemia normochromic normocytic (suspected bleeding dd chronic disease) and proevaluation due to prolonged hemostatic screening test (associated with severe sepsis without a sign of fulminant hepatic failure).

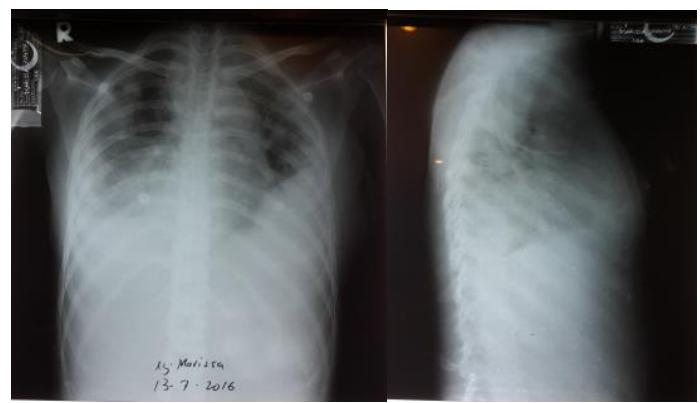

Figure 1. Bilateral pleural effusions and infiltrates in both lungs on July 13th, 2016

On July 14th, 2016, category 1 ATD (INH 200 mg, Rifampicin $450 \mathrm{mg}$, Pirazinamid $1000 \mathrm{mg}$, Ethambutol $750 \mathrm{mg}$ ) was provided to the patient without any additional symptom. The general condition of the patient overall improved with previously prescribed therapy and additional ATD.

Before receiving a pack of red blood cell (PRBC) transfusion, the patient was tested for blood smear on July 14th, 2016. The result was erythrocyte: majority hypochromic, anisopoikilocytosis (microcyte, normocyte, ferocyte, fragmentocyte), polychromasia cell $(+)$, normoblast $(-)$; leukocyte: increased in number, dominated by segment neutrophile, immature granulocytes (+), (Stab, metamyelocyte, myelocyte), blast (-); and thrombocyte: normal amount, giant platelet $(-)$, clumping platelet (-). Blood smear test impressed an anemia hypochromic anisopoikilocytosis with a shift to the left leukocytosis.

On July 15th, 2016, sputum acid-fast bacillus (AFB) smear test and gram smear test were performed. The results of these tests were BTA 2+ without any gram-positive or -negative bacteria. Chest ultrasonography with a marker on the same day in the operation chamber also showed organized bilateral pleural effusions.

\section{Complicated care stage}

On July 16th, 2016, the patient defecated a black and tarry stool in a considerable amount. The patient was seen pale while physical examination showed anemic conjunctiva. The patient was administered tranexamic acid 1 gram and vitamin $\mathrm{K}$ intravenously every 8 hours, omeprazole $40 \mathrm{mg}$ intravenously every six hours, and a tablespoon of oral sucralfate every 8 hours.

A laboratory study showed a worsen anemia from the previous test $(\mathrm{Hb} 7.5 \mathrm{~g} / \mathrm{dL})$ became $5.4 \mathrm{~g} / \mathrm{dL}$, although the patient had received 2 packs of PRBC. The thrombocyte level also decreased from $200000 / \mu \mathrm{L}$ to 90 $700 / \mu \mathrm{L}$, while the level of leukocytes and neutrophile showed an improvement.

The results of consultation from Department of Internal Medicine were: Melena/stress-related mucosal diseases and suspicion of autoimmune diseases or any immunocompromised condition due to multi pathology. The melena treatment was continued. The patient was suggested to be given 2 packs of PRBC until the $\mathrm{Hb}$ level was more than $10 \mathrm{~g} / \mathrm{dL}$. It was also recommended to install a nasogastric tube to evaluate gastric juice production, yet the patient and her family refused.

The underlying cause of melena and thrombocytopenia was evaluated and consulted to Division of Tropical Infection for any thrombocytopenia condition associated with drugs, which was given to the patient during treatment for CAP and pulmonary TB. It was suggested to discontinue Rifampicin as it was the most suspected to induce thrombocytopenia in the patient. The remaining therapy proceeded, and ATD regimen was provided without Rifampicin. 
Chest radiography on July 18th, 2016 (Figure 2) showed less infiltration in both lungs with organized bilateral pleural effusions. Complete blood count (CBC) on July 20th, 2016, showed improvement in leukocyte and thrombocytes as well as hemoglobin of the patient. The laboratory results were leukocytes $(15.2 \times 103 / \mathrm{uL})$, neutrophil $81.4 \%$, Hb $7.2 \mathrm{~g} / \mathrm{dL}$, and HCT $22.7 \%$, PLT $338 \times 103 /$ uL. Clinically, the patient had improved overall.

On July 22nd, 2016, the patient was taken for abdominal sonography that showed no abnormality in liver, spleen, pancreas, bladder, uterus, left and right adnexa, and left kidney, no lymph node enlargement in paraaortic area, while there was caliectasis in right kidney, organized right pleural effusion, and minimal pleural effusion that had partly organized.

CBC was also performed on July 23th, 2016 to evaluate $\mathrm{Hb}$ level following PRBC transfusion as well as thrombocyte level of the patient. Laboratory test showed improvements as follows: Leukocytes (18.9x103/uL), neutrophil $62.4 \%, \mathrm{Hb} 10.9 \mathrm{~g} / \mathrm{dL}, \mathrm{HCT} 34.8 \%$, and PLT $563 \times 103 / \mathrm{uL}$

Additional tests, such as aerobic culture and blood smear test, did not find any bacterial growth. Chest radiology on July $25^{\text {th }}, 2016$ showed there were bilateral organized pleural effusions (Figure 3 ).

\section{Outpatient stage}

Clinical

evaluation and Rifampicin desensitization were performed in DOTS outpatient clinic of Dr. Soetomo General Hospital. The patient was given Rifampicin gradually from $1 / 3$ dosage, raised slowly every three days to $2 / 3$ dosage, and finally, full dosage. $\mathrm{CBC}$ was tested in the desensitazation period to discover if thrombocytopenia occurred when Rifampicin was prescribed. The first densitazation was performed on August $2^{\text {nd }}, 2016$. The patient was given Rifampicin $150 \mathrm{mg}$ for three days consecutively, and the result of laboratory test on August $5^{\text {th }}, 2016$, was leucocytes $\left(6.51 \times 10^{3} / \mathrm{uL}\right)$, neutrophil $71.2 \%, \mathrm{Hb} 10.4 \mathrm{~g} / \mathrm{dL}, \mathrm{HCT}$ $32.8 \%$, PLT $362 \times 10^{3} / \mathrm{uL}$. There was no thrombocytopenia on $\mathrm{CBC}$ test and no symptom of any bleeding. Desensitazation proceeded with prescribed Rifampicin $300 \mathrm{mg}$ and then full dosage, $450 \mathrm{mg}$. On clinical evaluation, there was no sign of bleeding and

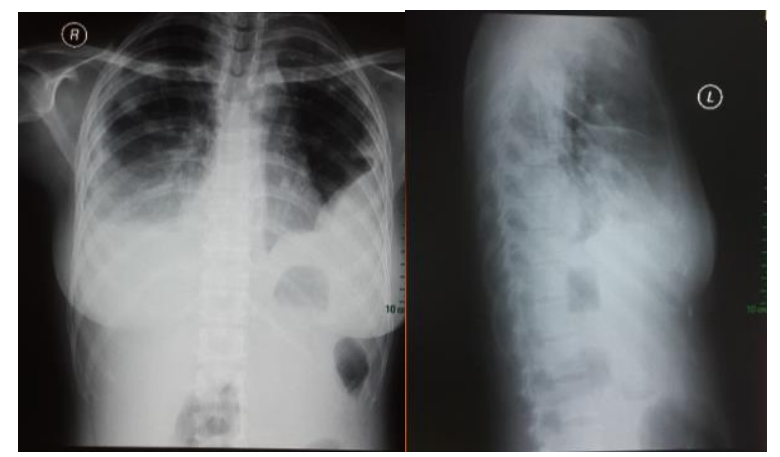

Figure 2. Chest radiography on July $18^{\text {th }}, 2016$ showed less infiltration in both lungs with organized bilateral pleural effusions laboratory test on August $10^{\text {th }} 2016$ showed normal result, leucocytes $\left(7.16 \times 10^{3} / \mathrm{uL}\right)$, neutrophil $76.98 \%, \mathrm{Hb}$ $10.8 \mathrm{~g} / \mathrm{dL}$, HCT $36.2 \%$, and PLT $308 \times 10^{3} / \mathrm{uL}$. When the patient was discharged, Levofloxacin was provided as Rifampicin replacement. However, because of full desensitization, Levofloxacin was discontinued, and Rifampicin was prescribed again as category one of ATD.

The patient continued her TB treatment in Community Health Care of Sepanjang. Currently, the patient has been in the fourth month of the continuation phase. AFB smear test evaluation at the end of the second month of the treatment showed a conversion to negative. There was no symptom of bleeding on the second phase, which was a phase consisted of Rifampicin and Isoniazid consumed thrice a week.

\section{DISCUSSION}

\section{Thrombocytopenia \\ Definition}

Thrombocytopenia is defined as a disorder, which showed an abnormality on the low amount of thrombocyte. Thrombocytopenia is classified mild if the amount was between $70-150 \times 10^{3}$ per uL $(70-150 \times$ $10^{9}$ per L), moderate if it was $20-70 \times 10^{3}$ per uL $(20 \times$ $10^{9}$ per L), and severe if less than $20 \times 10^{3}$ per uL $(20 \times$ $10^{9}$ per L). ${ }^{4}$

\section{Etiology}

Thrombocytopenia was commonly cofounded when Complete blood count (CBC) was performed. To date, the etiology is still not fully elucidated and need more additional examination. ${ }^{4}$

Thrombocytopenia could emerge because of decreased production of thrombocytes, increased platelet consumption, or sequestration. The systematical approach should be adopted to evaluate incidental thrombocytopenia. A physician must inquire history of the patients, any sign of melena, rash, fever, and bleeding. A physician should also seek for any kind of medication, immunization, transfusion, family history, and medical history. History of either acute or chronic alcohol consumption should be asked too. Patients in a long-term hospitalization should also be evaluated of any heparin administration, which will yield heparininduced thrombocytopenia. Pregnant woman with a symptom of sight, headache, abdominal pain, or flu-like symptom might be a sign of pre-eclampsia or HELLP syndrome (hemolysis, elevated liver enzymes, and low platelet count). ${ }^{4}$

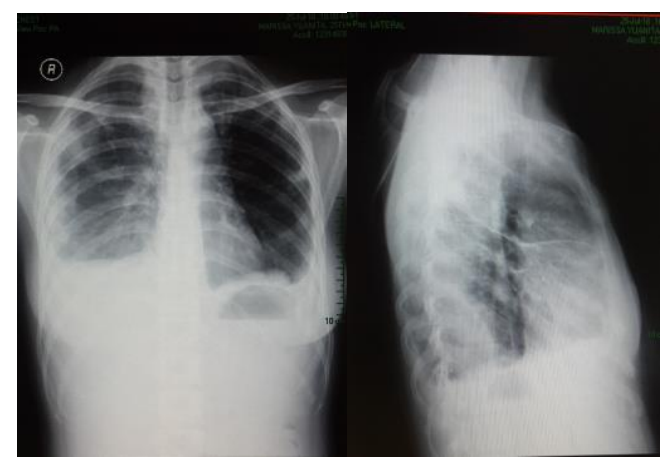

Figure 3. Chest radiology on July $25^{\text {th }}, 2016$ showed there were bilateral organized pleural effusions 
Table 1. Mechanism of DITP. ${ }^{4}$

\begin{tabular}{|c|c|c|c|}
\hline Classification & Mechanism & Incidence & Example of Drugs \\
\hline $\begin{array}{l}\text { Hapten-dependent } \\
\text { antibody }\end{array}$ & $\begin{array}{l}\text { Hapten links caovalently to } \\
\text { membrane protein and induces drug } \\
\text { specific immune response }\end{array}$ & Very rare & $\begin{array}{l}\text { Penicillin, possibly some } \\
\text { cephalosporin antibiotics }\end{array}$ \\
\hline Quinine-type drug & $\begin{array}{l}\text { Drug induces antibody that bunds to } \\
\text { membrane proteinin presence of } \\
\text { soluble drug }\end{array}$ & $\begin{array}{l}26 \text { cases per } 1 \text { million users of quinine } \\
\text { per week, probably fewer cases with } \\
\text { other drugs }\end{array}$ & $\begin{array}{l}\text { Quinine, sulfonamide } \\
\text { antibiotics, nonsteroidal } \\
\text { antiinflamatory drugs }\end{array}$ \\
\hline Fiban-type drug & $\begin{array}{l}\text { Drug reacts with glycoprotein IIb/IIa } \\
\text { to induce a comformational charge } \\
\text { (neoepitope) recorgnized by } \\
\text { antibody (not yet confirmed) }\end{array}$ & $0,2-0,5 \%$ & Tirofiban, eptifibatide \\
\hline Drug-specific antibody & $\begin{array}{l}\text { Antibody recognizes murine } \\
\text { component of chimeric Fab fragment } \\
\text { aaspecific for platelet membrane } \\
\text { glycoprotein IIIa }\end{array}$ & $\begin{array}{l}0,5-1,0 \% \text { after first exposure, } 10-14 \% \\
\text { after second exposure }\end{array}$ & Abciximab \\
\hline Autoantibody & $\begin{array}{l}\text { Drug induces antibody that reacts } \\
\text { with autologous platelets in absence } \\
\text { of drug }\end{array}$ & $\begin{array}{l}1,0 \% \text { with gold, very rare with } \\
\text { procainamide and other drugs }\end{array}$ & Gold salt, procainamide \\
\hline Immune complex & $\begin{array}{l}\text { Drug induces antibody factor } 4 \text {, } \\
\text { producing immune complex for } w\end{array}$ & $\begin{array}{l}3-6 \% \text { among patients treated with } \\
\text { unfractionated heparin for } 7 \text { days, rare } \\
\text { with low-molecular-weight heparin }\end{array}$ & Heparins \\
\hline
\end{tabular}

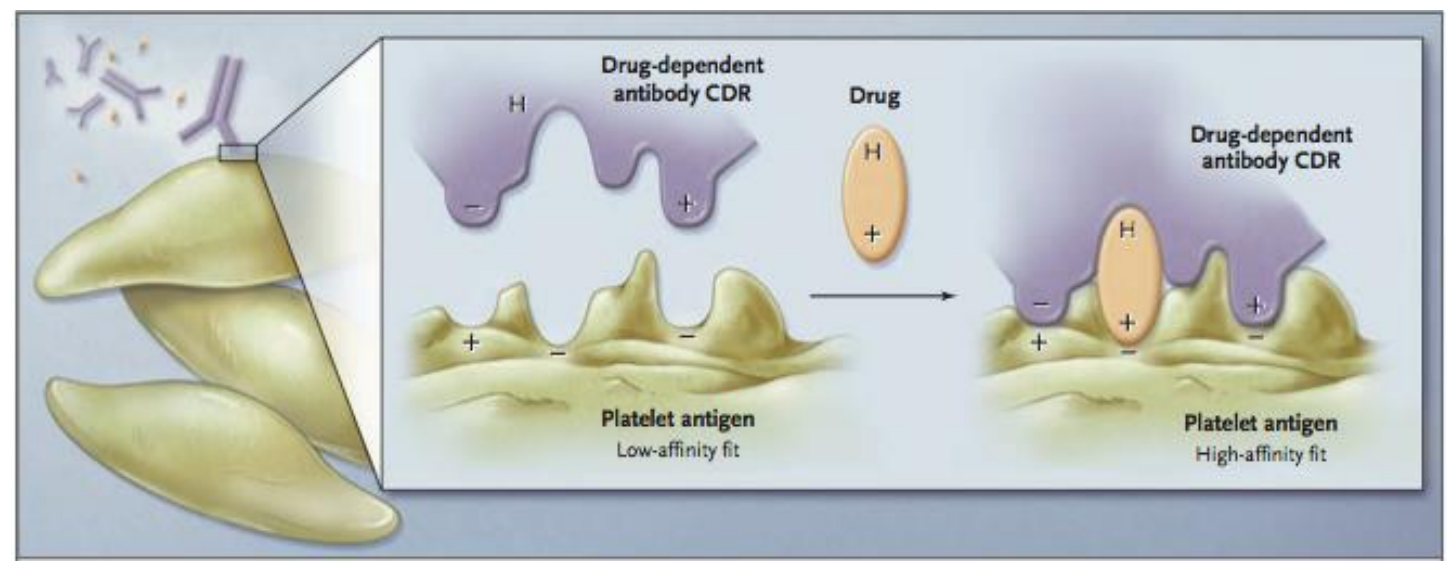

Figure 4. The binding model between drug-dependent antibody

The majority of the mechanism associated with thrombocytopenia is the immune, such as haptendependent antibody formation, drug-glycoprotein complex antibody formation, autoantibody formation, ligan-induced binding site creation, drug-specific antibody formation, and immune complex-mediated antibody formation (Table 1). Rifampicin was suspected as a hapten and produced antibodies following incorporation with other molecules on plasma. $^{5}$

A study by Karl Landsteiner in immunochemistry field in 1930 showed that a small molecule, such as drugs, organic compounds, peptides, and oligosaccharides, with molecular weight less than 2-5 $\mathrm{kDa}$ could not induce any immune response. Vice versa, these small molecules, which are called haptens, could produce an immune response when they are on covalently bound with their carrier protein. ${ }^{10}$

The antibody could be the underlying cause of the drug-dependent thrombocytopenia that reacts weakly with epitope from glycoprotein target (Figure 4). The binding ability $\left(\mathrm{K}_{\mathrm{A}}\right)$ to interact is too low to allow some molecular antibody binding in case of no drug (lowaffinity fit). The drug has some structural elements that complement the negative site of the glycoprotein and hydrophobic site $(\mathrm{H})$ of the complementaritydetermining region (CDR) of the antibody. The drug will interact with that site so that there will be a balance between both proteins while raising $\mathrm{K}_{\mathrm{A}}$ to a value, which will prompt binding between antibody, antigen, and drugs, that will be reached in the circulation after ingestion of the drugs (high-affinity fit). ${ }^{11}$ 
A study from Kassa, et al. showed that the amount of the thrombocytes was an essential hematology profile and presented a significant difference between prior TB treatment compared to 2 months following TB treatment $(p=0.010)$. Almost half of the TB patients, who had a high number of thrombocytes before treatment, showed a significant reduction after the intensive phase. This finding was also supported by a study from India that showed a decline in thrombocytes number that may be caused by the adverse effect of ATD and immune destruction from the thrombocytes. ${ }^{12}$

In the patient of this study, the suspicion as the underlying cause of the thrombocytopenia was mainly towards Rifampicin without ruling out the other agents that might also induce. In theory, Rifampicin could induce thrombocytopenia in two manners by acting as a hapten-dependent antibody or by a drug-dependent antibody.

\section{Other Agents}

More than 200 agents (including some herbal groups) have been reported inducing DITP. Aster and Bougie identified 85 agents that had been considered leading to thrombocytopenia. Some of them are agents that are commonly used in daily practice, such as heparin, quinine, antibiotics (Penicillin, Cephalosporin, Rifampicin, Vancomycin, and Sulfonamide), antiplatelet agents, Glycoprotein IIb/IIIa (GPIIb/IIIa) inhibitor (Tirofiban, Eptifibatide, Abciximab), antirheumatic agents (gold, Dpenicillamine), antiepileptic drugs (Natrium valproate, Phenytoin), antiarrhythmic (Amrinone), and novel biologic agents including Infliximab and Rituximab., ${ }^{9,11}$

During treatment, the patient was administered with antibiotic to treat acute infection and sepsis. In this case, Meropenem and Levofloxacin, antibiotics from beta-lactam group and fluoroquinolone group, respectively, were given to the patient. The patient was also prescribed with category one of ATD, which was Isoniazid, Rifampicin, Pyrazinamide, and Ethambutol. The suspicion towards underlying agents that lead to thrombocytopenia was mainly toward ATD even though other agents were not ruled out, such as an antibiotic, a group that could also lead to DITP. It was decided to discontinue Rifampicin while continuing other antibiotics, Meropenem and Levofloxacin. The role of Rifampicin in pulmonary TB treatment was temporarily replaced by Levofloxacin, which also served to treat acute infection in our patient.

\section{Clinical Presentation}

The patients usually present wide petechiae or purpura because of the low amount of the platelet in plasma (usually $<10 \times 10^{9} / \mathrm{L}$ ). Life-threatening bleeding including intracranial haemorrhage could occur in DITP. 8,9

In the patient of this study, an acute bleeding condition as melena was found that yielded more anemic condition than before, although she had been supported by PRBC transfusion. In $\mathrm{CBC}$, the patient received thrombocytopenia that might be caused by the adverse effect of a drug, which was consumed during the treatment. There was no history of bleeding in the patient.

Rifampicin, an antibiotic that widely used in TB regimen, has been suspected in the case of immune thrombocytopenia. DITP due to Rifampicin usually occurred after high dosage administration, thrice a week. IgG sensitization on targeted thrombocytes might happen by Rifampicin with the concentration lower than peak concentration following administration of Rifampicin $600 \mathrm{mg}$. It is still very few cases of Rifampicin-dependent anti-platelet antibodies that have been reported and the lack of laboratory documentation. $5,16,17$

DITP is an exclusion diagnosis, which is obtained by ruling out other underlying causes that resulted in thrombocytopenia. Therefore, its diagnosis is complex and often missed as other immunity disorders, such as immune thrombocytopenic purpura (ITP), posttransfusion purpura (PTP), or platelet transfusion refractoriness (PTR), particularly in patients who receive various kinds of drug. ${ }^{5,16,17}$

Clinical criteria (George criteria) had been established to implicate a drug, which had been suspected of inducing thrombocytopenia, as following:

1. Exposure of the candidate drug prior to thrombocytopenia.

2. Fully and sustainable improvement of thrombocytopenia after discontinuation of the candidate drug.

3. The suspected drug is the only drug used prior to thrombocytopenia, or if other agents were proceeded or reintroduced after discontinuation of the alleged agents, then the level of thrombocytes is normal and sustainable. Rule out other thrombocytopenia inducer.

4. Re-exposure with candidate drugs will result in refractory thrombocytopenia.

The evidence is considered valid if criteria 1,2,3, and 4 are met; Probable if criteria 1, 2, and 3 are fulfilled; Possible if only 1 criterion is fulfilled; and Unlikely if there is no fulfillment criteria.

In the patient of this study, there was no previous medication history for TB treatment. George criteria that were used to evaluate the cause, which was associated with DITP, the requirements were implemented and fulfilled for criteria 1,2, and 3 in the patient. Therefore, 
the patient could be characterized as probable. The fourth criterion from George was not met. It was because in the patient, after reintroducing Rifampicin gradually from $1 / 3$ to $2 / 3$ and finally full dosage, there was no repeated thrombocytopenia, and she could continue her treatment, which is currently in the continuous phase.

\section{Onset of DITP}

The average onset of thrombocytopenia occurred 1-2 weeks following the exposure of the suspected drug. However, the onset itself could emerge broadly from a few hours to years, depending on if the patient has already had the antibody, which is needed to bind an amnestic response. It is mainly because of the low titer of antibody that is produced

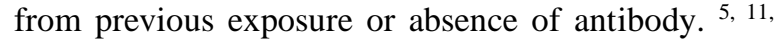
16,17

In a case report by Reksa, et al., the patient had thrombocytopenia for the first time three weeks following the suspected drug, although there was a previous exposure ten years before. In a presentation that occurred one hour after exposure, it was highly suggestive as an amnestic response. This case study emphasized the importance of continuous monitoring at the patient, who received ATD, because all of ATD agents could induce severe thrombocytopenia anytime during treatment. ${ }^{18,19}$

The patient in this study showed thrombocytopenia on day 4 during therapy in the hospital. The patient showed bleeding manifestation as melena with the level of thrombocytes as low as 90.700. Then, she was diagnosed as DITP clinically, and the agents, which mostly related to DITP, were evaluated. The patient was admitted with infection and sepsis condition. Yet, during four days of treatment, there was an improvement in clinical and laboratory presentation. Hence, the possibility of thrombocytopenia induced by other comorbid factors, including infection, could be ruled out.

\section{Additional Examination}

The diagnosis of DITP is often empirically. A patient, who suffers thrombocytopenia because of the use of a single drug, is usually improved after discontinuation of the agents. It implicates that the drug induces thrombocytopenia. There are various methods to detect any drug-dependent antibodies (DDAbs). Those methods include radio-labeled or fluorescein-labeled (platelet immunofluorescence test) anti-IgG to detect platelet-bound immunoglobulin, enzyme-linked immunosorbent assay (ELISA), flow cytometry, and immunoprecipitation-Western blotting (IP-WB). ELISA and IP-WB have a better sensitivity to assess any DDAbs. The reaction is assessed by comparing serum reactivity or sample plasma in a condition with or without the drug. Flow cytometry is a fast and sensitive technique to evaluate any plateletreactive antibody, which is induced by some agents. ELISA method is insensitive in identifying target molecules where DDAbs react. This includes antigen
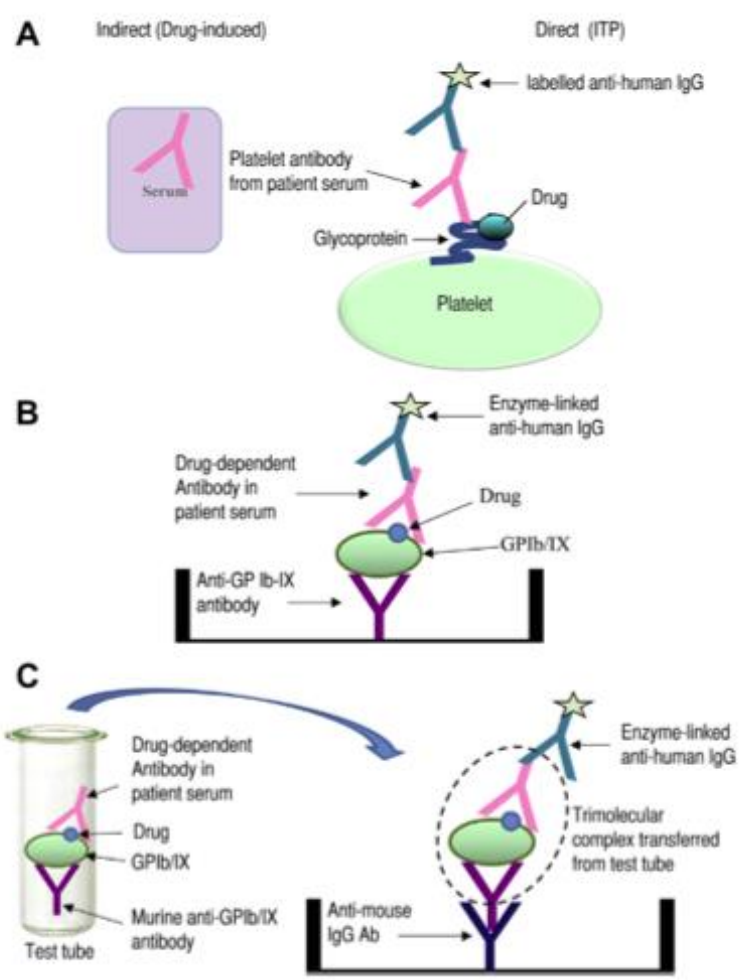

Figure 5. Antiplatelet antibody assays. (A) Platelet associated $\mathrm{IgG}(\mathrm{PA}-\mathrm{IgG})$ : direct and indirect binding assay. (B) Modified antigen-capture enzyme-linked immunoadsorbent assay. (C) Monoclonal antibody-specific immobilization of platelet antigen assay. ${ }^{9}$

capture ELISA assay (ACE, Maipa) and modified antigen capture ELISA. ${ }^{10,20}$

Various factors should be considered for any failure to detect DDAbs, such as low solubility of some agents in liquid media; a possibility that the sensitized agents could become a structured and modified model from drug susceptibility that is resulted from in vivo metabolism; and maybe the autologous cells are used for the test. ${ }^{10,20}$

The binding site for Rifampicin dependent antibody ( $\mathrm{rd}-\mathrm{Ab})$ is located on the platelet membrane glycoprotein complex (GP) Ib/IX. It is reported in a patient that $\mathrm{rd}-\mathrm{Ab}$ induced thrombocytopenia by binding the platelet GPIIb/IIIa complex. This was suggested by flow cytometry, a monoclonal antibody (mAb), MAIPA assay, and immunoprecipitation method. The result showed that rd-Ab was derived from $\mathrm{IgG}$ class, not from IgM class. Rd-Ab IgG was bound with GPIIb/IIIa complex on the platelet membrane with Rifampicin concentration as low as $6 \mathrm{lg} / \mathrm{ml}$., which was a very low concentration compared to peak plasma concentration of Rifampicin after administration standard dosage of Rifampicin 600 mg. ${ }^{5,16}$

\section{Drug-induced Thrombocytopenia Management Drug discontinuation and replacement}

Physician must consider ceasing any suspected agents that induce thrombocytopenia and its alternative 
management to resolve thrombocytopenia. The bleeding will be more severe, and the level of thrombocytes will continue to drop if the suspected agents are not ceased. ${ }^{18,19}$ It is expected that there is no cross-reactivity between different structural agents from the same class or between stereoisomer because of the high exclusivity of the reaction. Hence, the drug from the same class could be replaced. On platelet aggregation reaction, thrombocytopenia is usually mild. However, evaluation of thrombocyte level is necessary. If the condition is severe $\left(<50 \times 10^{3} / \mathrm{mm}^{3}\right)$ or the patients suffer any bleeding, the suspected agents must be discontinued. ${ }^{14,15,20}$

\section{Anti-tuberculosis drug desensitization}

The first line of ATD consisted of Isoniazid, Rifampicin, Ethambutol, and Pyrazinamide. These drugs are associated with adverse drug reaction (ADR), which has yielded a reduction in success rate on active TB patients who are sensitive to ATD. ADRs that were usually reported from the first-line ATD are rash, pruritus, hepatitis, nausea vomiting, thrombocytopenia, flu-like symptom, arthralgia, and neuropsychiatric symptom..$^{20,21}$ ADR could result in significant morbidity, which will lead to first-line ATD withdrawal and substitution with the second line of ATD that is less effective and tolerate worse agents. This substitution will prolong treatment duration and may influence regimen adherence. Severe ADR could result in increased hospitalization, and for outpatient settings, it will lead to more visitation. Prediction of who has an increased risk for ADR of first-line TB treatment could help in identifying the patient that needed more caution evaluation, so that it could prevent morbidity potential, hospitalization, and mortality. Female, aged above 60 years old, Asian, and has human immunodeficiency virus (HIV) have been associated with increased ADR incidence on first-line TB treatment. History of hepatitis B or C had also been related to the development of severe ADR of the first-line TB treatment, although it needed more confirmation studies. ${ }^{21,22}$

There is no better alternative option for firstline ATD in TB treatment. However, hypersensitivity and allergy towards these drugs are not infrequent. Limitations in diagnostic tests either in vitro or in vivo to discover the suspected TB agents that lead to allergy are often needed desensitization-rechallenge (D-R) adjustment regimen to reintroduce the appropriate TB treatment. ${ }^{21,23}$

\section{Rifampicin}

Rifampicin is an active antituberculosis bactericidal that is very active towards rapidly developed extracellular organisms while also having activity on intracellular bactericidal. Rifampicin also has activity towards slowly developed bacterium, such as mycobacterium tuberculosis. Rifampicin inhibits DNA-dependent RNA polymerase of cells. From the pharmacokinetic aspect of Rifampicin, this agent is easily absorbed from the gastrointestinal tract. Peak serum about $10 \mu \mathrm{g} / \mathrm{ml}$ is achieved about two until four hours after administration with $10 \mathrm{mg} / \mathrm{kgBB}$ dosage on an empty stomach. Rifampicin absorption will be reduced if it is consumed together with a meal. The pharmacokinetic, either oral or intravenous in the pediatric population, is similar as in adults. In a normal subject, the average half-life of Rifampicin in serum was 3 hours following $600 \mathrm{mg}$ administration, while it would increase to 5.1 hours after $900 \mathrm{mg}$ administration. With repeated consumption, the half-life will be reduced, and the agent will peak about 2-3 hours. With $600 \mathrm{mg} /$ day dosage, the effect will not be different on a patient with kidney failure, and there is no obligation to adjust the dosage. Rifampicin is swiftly eliminated in the bile, then there will be enterohepatic circulation. During this process, Rifampicin will be deacylated progressively so that almost all Rifampicin in this form will be in the bile after about 6 hours. Reabsorption in the intestine will be reduced with acetylation and elimination. About $30 \%$ of the drug will be excreted in the urine, while half of them shifting in form. Rifampicin is widely distributed to the whole body. The effective concentration of Rifampicin is achieved in many organs and body fluid, including cerebrospinal fluid. Approximately $80 \%$ of Rifampicin is bound with protein. Majority of protein-bound Rifampicin is not ionized, so that it could be distributed freely on the tissue. ${ }^{22}$

The patient of this study was initially provided first-line standard TB regimen in the first four days of treatment. Then, there was melena, and the laboratory test showed a drop in hemoglobin and thrombocyte levels. It was decided to withdraw Rifampicin from her regimen and replaced by Levofloxacin administration $750 \mathrm{mg}$ intravenously for every 24 hours while it was also serving as pneumonia treatment in the patient.

Furthermore, the patient was given Rifampicin desensitization in DOTS outpatient clinic of Dr. Soetomo General Hospital. The first prescribed Rifampicin dosage was $1 / 3$. Then, it was raised every three days until the full dosage was achieved. CBC monitoring was performed during sensitization. From desensitization, it could be inferred that the patient was able to continue with a full dosage Rifampicin and currently on the fourth month of continuous phase without any thrombocytopenia or any bleeding manifestation.

\section{CONCLUSION}

Even though it is often complicated by various potential etiology of thrombocytopenia, the suspected 
drug isolation and cessation on a patient in acute condition must be adequately performed and organized, as if the treatment continues, it could lead to fatality. On the other hand, restricting some primary suspected agents could lead to a medical treatment limitation. The clinical challenge in druginduced thrombocytopenia is identifying the suspected agent as fast and as early as the disorder occurs so that the right management could be started while hindering bleeding complications. A thorough and clear medication history could help in establishing the temporal association. At the same time, initial examination, dropping level, and an absolute amount of thrombocytes are helping to isolate the underlying cause of DITP. There are many available novel technologies to detect drug-dependent plateletreactive antibodies on immune thrombocytopenia patients.

\section{REFERENCES}

1. Efferen LS. Tuberculosis and pregnancy. Current opinion in pulmonary medicine. 2007 May 1;13(3):205-11.

2. Verma AK, Singh A, Chandra A, Kumar S, Gupta RK. Rifampicin-induced thrombocytopenia. Indian journal of pharmacology. $2010 \mathrm{Jul}$ 1;42(4):240.

3. Garg R, Gupta N, Mehra S, Singh R, Prasad R. Rifampicin induced thrombocytopenia. INDIAN JOURNAL OF TUBERCULOSIS. 2007 Apr 20;54(2):94.

4. Gauer RL, Braun MM. Thrombocytopenia. American family physician. 2012 Mar 15;85(6).

5. Pereira J, Hidalgo $\mathrm{P}$, Ocqueteau $\mathrm{M}$, Blacutt $\mathrm{M}$, Marchesse M, Nien Y, Letelier L, Mezzano D. Glycoprotein Ib/IX complex is the target in Rifampicin-induced immune thrombocytopenia. British journal of haematology. 2000 Sep 1;110(4):907-10.

6. Aster RH, Curtis BR, McFarland JG, Bougie DW. Drug-induced immune thrombocytopenia: pathogenesis, diagnosis, and management. Journal of Thrombosis and Haemostasis. 2009 Jun 1;7(6):911-8.

7. Konkle B. Disorder of platelets and vessel wall: Harisson's Principle of Interna Medicine. 2009; 115: 9659.

8. Kassa E, Enawgaw B, Gelaw A, Gelaw B. Effect of antituberculosis drugs on hematological profiles of tuberculosis patients attending at University of Gondar Hospital, Northwest Ethiopia. BMC hematology. 2016 Jan 8;16(1):1

9. Chong BH, Choi PY, Khachigian L, Perdomo J. Druginduced Immune Thrombocytopenia. Hematol Oncol Clin N Am. 2013; 27: 521-540.

10. Visentin GP, Liu CY. Drug-induced thrombocytopenia. Hematology/oncology clinics of North America. 2007 Aug 31;21(4):685-96.

11. Aster RH, Bougie DW. Drug-induced immune thrombo- cytopenia. New England Journal of Medicine. 2007 Aug 9;357(6):580-7.

12. Kolyva AS, Karakousis PC. Old and new TB drugs: Mechanisms of action and resistance. INTECH Open Access Publisher; 2012.

13. Goyette RE, Key NS, Ely EW. Hematologic changes in sepsis and their therapeutic implications. InSeminars in respiratory and critical care medicine 2004 Dec (Vol. 25, No. 06, pp. 645-659). Copyright@ 2004 by Thieme Medical Publishers, Inc., 333 Seventh Avenue, New York, NY 10001, USA.

14. Van den Bemt PM, Meyboom RH, Egberts AC. Druginduced immune thrombocytopenia. Drug safety. 2004 Dec 1;27(15):1243-52.

15. Wazny LD, Ariano RE. Evaluation and Management of Drug-Induced Thrombocytopenia in the Acutely Ill Patient. Pharmacotherapy: The Journal of Human Pharmacology and Drug Therapy. 2000 Mar 1;20(3):292-307.

16. Burgess JK, Lopez JA, Gaudry LE, Chong BH. Rifampicin-dependent antibodies bind a similar or identical epitope to glycoprotein IX-specific quininedependent antibodies. Blood. 2000 Mar 15;95(6):198892.

17. Sun P, Wu G, Gao RL, Liu S, Phillips WD, Liang SX. Rifampicin-dependent antibodies target glycoprotein $\mathrm{IIb} / \mathrm{IIIa}$ and cause clearance of human platelets in NOD/SCID mice. British journal of haematology. 2016 Jan 1;172(1):137-40.

18. Bansal R, Sharma PK, Sharma A. A case of thrombocytopenia caused by Rifampicin and pyrazinamide. Indian journal of pharmacology. 2013 July 1;45(4):405.

19. Rekha VB, Adhilakshmi AR, Jawahar MS. Rifampicininduced acute thrombocytopenia. Lung India. 2005 Oct $1 ; 22(4): 122$

20. Arnold DM, Nazi I, Smith JW, Warkentin TE Management of immune-mediated thrombocytopenia. Rossi's Principles of Transfusion Medicine. 2016:245-64.

21. Kementerian Kesehatan Republik Indonesia. Pedoman Nasional pengendalian Tuberkulosis. Jakarta 2014.

22. Marra F, Marra CA, Bruchet N, Richardson K, Moadebi S, Elwood RK, FitzGerald JM. Adverse drug reactions associated with first-line anti-tuberculosis drug regimens. The International Journal of Tuberculosis and Lung Disease. 2007 Aug 1;11(8):868-75.

23. Thong BY, Chia FL, Tan SC, Tan TC, Leong KP, Tan JW, Tang CY, Hou JF, Chan GY, Chng HH. A retrospective study on sequential desensitizationrechallenge for antituberculosis drug allergy. Asia Pacific Allergy. 2014 Jul;4(3):156.

24. Conde MB, Lapa e Silva JR. New regimens for reducing the duration of treatment of drug-susceptible pulmonary tuberculosis. Drug development research. 2011 Sep 1;72(6):501-8.

25. $\mathrm{Hu} \mathrm{Y}$, Coates AR, Mitchison DA. Sterilizing activities of fluoroquinolones against rifampin-tolerant populations of Mycobacterium tuberculosis. Antimicrobial agents and chemotherapy. 2003 Feb 1;47(2):653-7. 
